\title{
Synergetic Potential of Combination Probiotic Complex with Phytopharmaceuticals in Valproic Acid Induced Autism: Prenatal Model
}

\author{
Katta Sunand $^{* 1,2}$, G. Krishna Mohan ${ }^{1}$, Vasudha Bakshi ${ }^{2}$ \\ ${ }^{1}$ Centre for Pharmaceutical Sciences, Institute of Science \& Technology, JNTU Hyderabad, India. \\ ${ }^{2}$ Department of Pharmacology, Anurag University, Hyderabad, Andhra Pradesh, India.
}

\begin{abstract}
Background: Autism spectrum disorder (ASD) is a developing neuronal disorder with hindered social correspondence and repetitive behaviors. Hereditary and ecological factors have been related to this problem, including the introduction of valproic acid during pregnancy. Dysbiosis is the most common comorbid condition in autism, numerous probiotic strains have been known to forestall social impairments in autism.

Method: Prenatal autism model was done on $12^{\text {th }}$ embryonic day of pregnancy in Wistar rats with the valproic acid dose $400 \mathrm{mg} / \mathrm{kg}$, i.p. Those offspring exhibiting autistic symptoms were selected for study and were treated with polyphenols, probiotics and their combination for the study period of postnatal day (PND) 08-50.

Results: Prenatal model showed significant autistic and dysbiotic symptoms in the animals. With the probiotic and polyphenols treatment combination it was understood that bio-activation of polyphenols are crucial in the correction of neurochemical abnormality, oxidative stress, behavioral deficits and neuroprotection. Probiotic complex with acetyl-I-carnitine combination was also essential for the growth of gut bacteria, functionality of brain. Through this combination study behavioral and biochemical alterations were recovered.
\end{abstract}

Conclusion: Establishing a good ecosystem in the gut with good probiotics and polyphenols-like compounds is essential, gut targeted brain functionality is a remarkable way to challenge autistic disorder, advice on this combination may be helpful in the right way of accessing the gut-brain-axis and useful in amending of autism.

Keywords: Autism, Curcumin, Gut microbiota, Polyphenols, Valproic acid.

\section{INTRODUCTION}

The human microbiome encompasses multiple genomes of symbiotic microbiota such as bacteria, inhabiting the human body. ${ }^{1}$ Prospective association of many diseases with microbiotic behavior is currently being investigated for resolving the disease conditions through positively altering the microbiota. ${ }^{2}$

As indicated by the Diagnostic and Statistical Manual of Mental Disorders (DSM-5), children and individuals experiencing issues in communicating with others, limited interests or impaired interaction and repetitive behaviors that affect the capacity to work appropriately have been recognized with a neurodevelopmental condition identified as Autism Spectrum Disorder (ASD).3 Globally, it was recorded that 1 in 160 children are being diagnosed with ASD.4 ASD begins during early childhood with major altered social behaviors in the subject. ${ }^{5,6}$

Among numerous different causes, research has reported about $70 \%$ of autistic individuals having gastro-intestinal (GI) related issues like diarrhea, constipation and inflammatory bowel disease, playing a significant role in the advancement of autism. An association between precise gut health and reduction of symptoms in autism has been reported in patients as well. ${ }^{7-9}$ Specifically, valproic acid (VPA) when administered during pregnancy has a higher risk of developing $\mathrm{ASD}^{10,11}$

At present, there is no single best treatment known for ASD. As a matter of concern, the emerging attention is given to microbiota gut-brain-axis with an aim in improving conditions of ASD. Recent studies have bestowed a hope to improve autism through the healing advantages

\section{Corresponding author}

Katta Sunand

Email : sunandpharmacy@cvsr.ac.in 
of various herbal molecules and probiotic strains with fewer side effects. ${ }^{12}$

Polyphenols are organic chemicals utilized in curing neurodevelopmental disorders, by safeguarding neurons against injury. Researches have demonstrated that polyphenols, such as resveratrol, can control mitochondrial dysfunction associated with ASD patients. ${ }^{13}$ Curcumin re-established psychological manifestations by destroying reactive oxygen species (ROS) and inflammatory mediators in autism in rats. ${ }^{14}$

Amino acid such as acetyl-l-carnitine is also essential for energy production in gut bacteria, which can be helpful for the growth and production of metabolites (SCFA) in bacteria. Acetyl-1-carnitine also crosses BBB helps to energize the brain, brain and nerve support and also provide substrate for synthesis of acetyl choline in the brain. Amino acid such as acetyl-1-carnitine improves the psychological symptoms in animal models and shows gainful impacts in other behavioral abnormalities such as irritability, anxiety and mental function. ${ }^{15}$

A large number of probiotics have also been analyzed for their potential effects on gut-brain-axis in animal and human models. Lactobacillus strains exert a neuroprotective effect with the recovery of psychological behavior associated with gut microbiota remodeling in traumatic brain-injured mice. ${ }^{16}$ Treatment with Bifidobacteria strains attenuated depression and anxiety behaviors in anxious animals and significantly improved the immune system. ${ }^{17,18}$ Consumption of Streptococcus thermophilus has benefits in the management of many neurological disorders. ${ }^{19}$ Saccharomyces boulardii exerts therapeutic effects in a wide range of neurologic conditions including autism. ${ }^{20}$

Hence, the current study was focused on the use of polyphenols in combination with probiotics for the restitutionof ASD symptoms, by targeting gut-brain-axis in valproic acid-induced autism in rats.

\section{MATERIALS AND METHODS}

\subsection{Chemicals}

Probiotic strains (Bifidobacterium longum-UBBL-64, Bifidobacterium bifidum-UBBB-55(MTCC 5398), Lactobacillus rhamnosus-UBLR-58 (MTCC 5402), Streptococcus thermophilus-UBST-50 (MTCC 5382), Saccharomyces boulardii-28 obtained from Unique Biotech Ltd., Hyderabad, India; Inulin obtained from Natures Velvet Life care (India); Acetyl-L-Carnitine (Alcar) - West-Coast Pharmaceutical Works Ltd, Gujarat, India; Resveratrol - Sami labs, Hyderabad. India; Curcumin capsules were obtained from Himalaya Pharmaceuticals (India); Sodium Valproate was acquired from Sun Pharmaceuticals, Hyderabad (India); ELISA Kits for IL-6, TNF- $\alpha$, BDNF, 5-HT and AChE was obtained from Elab Sciences (India).

\subsection{Experimental Animals}

Female Wistar rats were procured from the National Institute of Nutrition, Hyderabad, Andhra Pradesh, India. Females with controlled fertility cycles, were mated overnight, and the morning when spermatozoa were found was designated as the first day of gestation. The experimental protocol was permitted by Institutional Animal Ethics Committee (IAEC) and care of the animals was done according to the guidelines of CPCSEA (Protocol No: I/IAEC/AGI/019/2018 WR ๑). Animals were kept up on standard laboratory pellet diet with water ad libitum. They were maintained in polypropylene cages and held under standard conditions of $12 \mathrm{hr}$ light/dark cycle, $23-25^{\circ} \mathrm{C}$ temperature and $35-60 \%$ humidity.

\subsection{Experimental Design}

The dose for curcumin, acetyl-1-carnitine and resveratrol was selected based on previous studies. ${ }^{21-23}$ During pregnancy, on embryonic day (ED) 12 , VPA was administered at a dosage of $400 \mathrm{mg} / \mathrm{kg}$, i.p. ${ }^{24}$ The well-being and weight of pregnant rats were examined recurrently up to delivery. The day on which pups were delivered was recorded as postnatal day (PND) 0 . On the $8^{\text {th }}$ postnatal day, male pups were divided into 8 groups, each group with eight pups $(n=8)$ namely,

Group I Vehicle treated group (Inulin 3mg, p.o daily) Group II Autistic Group (VPA $400 \mathrm{mg} / \mathrm{kg}$, i.p)

Group III Treatment with Probiotic Complex (NLT 1Billion CFU/ml, p.o)

Group IV Treatment with Curcumin (100 mg/kg, p.o) Group V Treatment with Acetyl-l-carnitine (200 mg/ $\mathrm{kg}$, p.o)

Group VI Treatment with Resveratrol (20 mg/kg, p.o) Group VII Treatment with Probiotic Complex + Curcumin (50 mg/kg, p.o)

Group VIII Treatment with Probiotic Complex + Acetyll-carnitine (100 mg/kg, p.o)

Group IX Treatment with Probiotic Complex + Resveratrol (10 mg/kg, p.o)

Probiotic complex was prepared with mixing of equal proportions of Lactobacillus acidophilus, Lactobacillus rhamnosus, Bifidobacteria longum, Bifidobacteria bifidum, S. thermophiles and Saccharomyces boulardii strains to the Inulin vehicle. The treatment duration for this study was 08-50 PND days with daily supplementation of probiotic strains not less than (NLT) 1 Billion colony forming units (CFU)/ $\mathrm{mL}$, p.o with the guidelines of administration and storage details provided by Unique Biotech Ltd. Further curcumin, acetyl-1-carnitine and resveratrol were mixed with Inulin vehicle and administer via oral route. Animals were exposed to various behavioral testing such as eyeopening, T-maze test, negative geotaxis, morris water 
maze test and social interaction studies. These behavioral tests were performed on different postnatal days up to PND 50 with the directions of standard protocol.

\subsection{Behavioral Studies}

\subsubsection{Eye Opening}

Eye-opening was observed consistently every day after birth from PND 12-16. Eye openings were recorded and ranked 0 when both eyes closed; 1 when only one eye opens and other closed and 2 for both eyes opened. ${ }^{25}$

\subsubsection{T-maze Test}

To assess repetitive and restricted behavior, T-maze is used. It is a spontaneous alteration test, which was performed on PND 29-31. Five trials were executed for each animal. For each trial, the primary choice of the rodent in the free decision arms was surveyed i.e., whether or not the rat at first entered the left or right arms. The limitation examined was the degree of alterations between the left and a right arm, which was continual, assessed compared to the arm visited in the past meeting. This model relies upon the characteristic proclivity of rodents to switch to and fro between the visited target arms in each trail over a movement of progressive trails. In this manner, a more elevated level of change between the arms was measured as typical rat behavior, while fewer alterations demonstrated psychological rigidity and repeated behavior. These data have been scored as; 0 for no alterations (consistently visiting similar arm for each of the five sessions), 1 for one alteration, 2 for two alterations, 3 for three alterations, and 4 for four alterations. ${ }^{26}$

\subsubsection{Negative Geotaxis}

Negative geotaxis was performed in an environment under regulated temperature by setting the mouse face down along a $45^{\circ}$ slope. Inactivity to rotate $180^{\circ}$ to such an extent that the head was mounted along the slope was noted with a limit of 30 seconds for each trial. ${ }^{27}$

\subsubsection{Morris Water Maze Test}

Morris water maze apparatus was used to evaluate the memory and this test was performed on PNDs 48-50. The aquarium was made up of 4 quadrants demonstrated by various hues for visual clues, it was loaded up with normal water and covered with milk, and a removable hidden stage was put at one quadrant. Each animal was placed and the escape latency period was recorded and repeated the same for next day PND 49. On PND 50, the platform was evacuated and an escape latency period was observed. $^{26}$

\subsubsection{Social Interaction Test}

Social interaction test was conducted on PND 36-40. Before the experiment, the animals were isolated and housed separately overnight. Two animals from the identical group, but diverse litters were placed into a circular cage provided by ambient light and temperature for 20 minutes. Frequency of the parameters such as allogrooming, anogenital inspections, pinning's, play behavior and social exploration was assessed. ${ }^{28}$

\subsection{Biochemical Parameters}

Rat brains were isolated and cleaned with ice-cold phosphate buffer (0.1M; pH: 7.4) to clear blood from brain. The brain was homogenized with phosphate buffer saline solution. The obtained supernatant was utilized for biochemical estimations of interleukin-6 (IL-6), tumor necrosis factor-alpha (TNF- $\alpha)$, 5- hydroxytryptamine (5-HT), brain derived neurotrophic factor (BDNF) and acetylcholinesterase (AChE).

\subsubsection{Estimation of IL-6, TNF- a, BDNF and 5-HT levels}

The estimation of IL-6, TNF- $\alpha$, BDNF and 5-HT were performed according to the instructions of ELISA kits procured from Rhetoric Life Sciences Pvt. Ltd., Bangalore, India.

\subsubsection{Estimation of Acetylcholinesterase (AChE) Activity}

The AChE concentration in the sample was estimated in brain tissue by the response of thiocholine with dithiobisnitrobenzoate ions. The pace of development of thiocholine from acetylcholine iodide was estimated utilizing a spectrophotometer at $412 \mathrm{~nm} .^{29}$

\subsection{Histopathology}

The isolated brains were placed in 10\% neutral formalin solution, processed and embedded in paraffin. Sagittal sections of the cerebellum ( $5 \mu \mathrm{m}$ thick) were stained with haematoxylin and eosin (H\&E) and analyzed using a light microscope for changes in the cerebellum. ${ }^{30}$

\subsection{Statistical Analysis}

All data are presented as Mean \pm S.E.M. The significance of difference among the groups was assessed using a one-way analysis of variance (ANOVA) followed by Kolmogorov-Smirnov test, Bonferroni's Multiple Comparison Test using GraphPad Prism 5 software and $P<0.05$ was considered significant.

\section{RESULTS}

\subsection{Behavioral Parameters}

\subsubsection{Effect of Probiotic Supplementation on Opening of Eye}

A significant $(P<0.001)$ delayed opening of the eye was observed in the autistic group on 12-16 PND when 
compared with the vehicle group. Treatment with polyphenols alone, probiotic complex and combination of polyphenols with probiotic complex has shown a significant $(P<0.001)$ improvement in opening of eye when compared with the autistic group. Among the polyphenols, a good score was observed in animals treated with resveratrol alone and among the combination, probiotic complex with resveratrol has shown highly significant improvement (Fig. 1).

\subsubsection{Effect of Probiotic Supplementation on T-Maze Test}

There was a significantly $(P<0.001)$ decreased alteration score observed in the autistic group by the T-maze test on 29-31 PND, when compared with the vehicle group. Treatment with polyphenols alone, probiotic complex and combination of polyphenols with probiotic complex has shown a significant $(P<0.001)$ improved alteration score, when compared with autistic group. Among the polyphenols, an enhanced score was performed by the animals treated with resveratrol alone and among the combination, probiotic complex with resveratrol has shown highly significant score (Fig. 2).

\subsubsection{Effect of Probiotic Supplementation on Negative Geotaxis}

There was a significant $(P<0.001)$ increase in time taken to re-orient along the inclined plane in the autistic group was reported on 9-21 PND, when compared to vehicle group. Treatment with polyphenols alone, probiotic complex and combination of polyphenols with probiotic complex has significantly $(P<0.001)$ decreased the time taken to re-orient, when compared with the autistic group. Among the polyphenols, a minimum reorient activity was observed in the animals treated with curcumin alone and among the combination; probiotic complex with curcumin has shown highly significant re-orient activity (Table 1).

\subsubsection{Effect of Probiotic Supplementation on Morris Water Maze}

Morris water maze test is used to evaluate the mental capability in subjects. There was significantly $(P<0.001)$ poor perception was observed in the autistic group as the latency to identify the hidden platform was delayed on 48-50 PND, when compared with the vehicle group. Treatment with polyphenols alone, probiotic complex and combination of polyphenols with probiotic complex has significantly $(P<0.001)$ increased the latency to identify the hidden platform when compared to the autistic subjects. Among the polyphenols, a minimum latency for identification was observed in the animals treated with acetyl-L-carnitine alone and among the combination, probiotic complex with acetyl-1-carnitine has shown highly significant result (Table 2).

\subsubsection{Effect of Probiotic Supplementation on Social Interaction}

Social interaction test was employed to assess the different patterns of behavior and communication. In the autistic group, there was a significantly $(P<0.001)$ decreased level of social interaction observed when compared to the

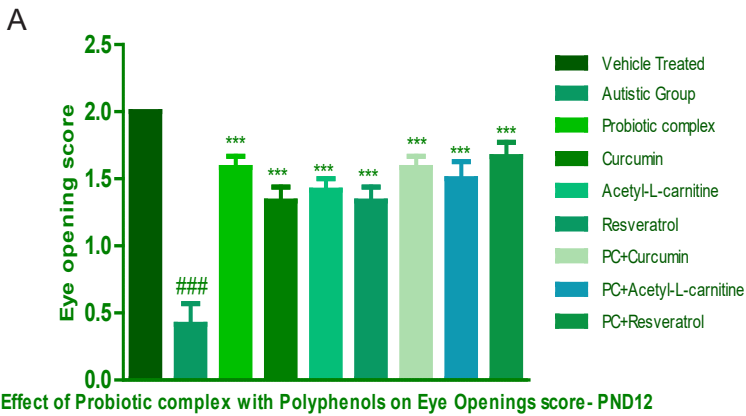

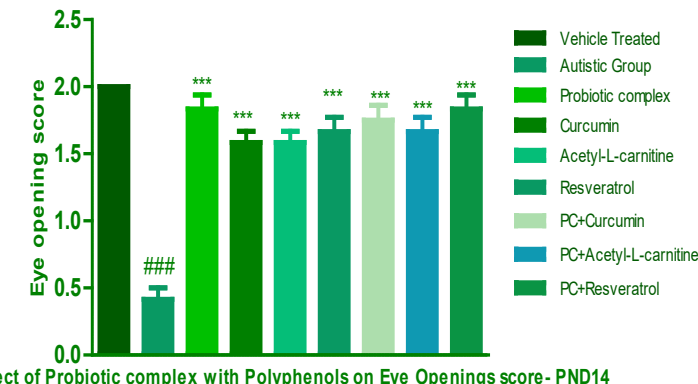

C

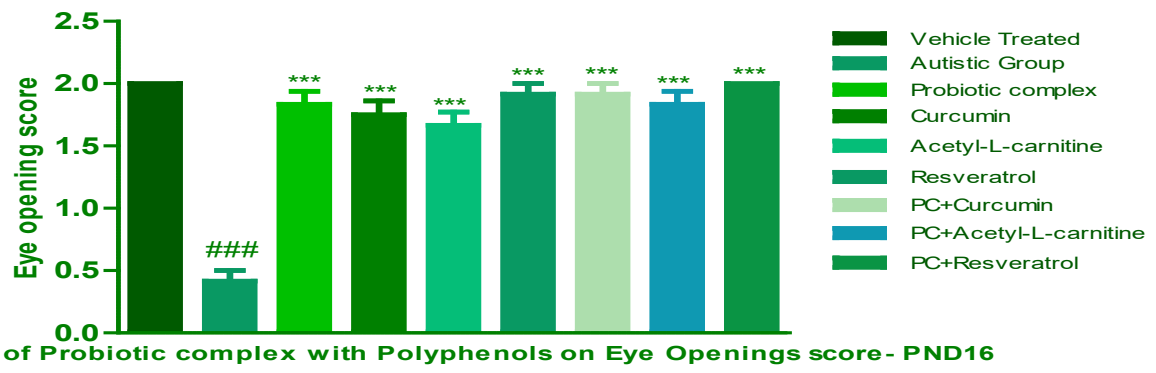

Fig. 1: Effect of probiotic complex with polyphenols combination on eye openings A) PND 12. (B) PND 14. (C) PND 16 were

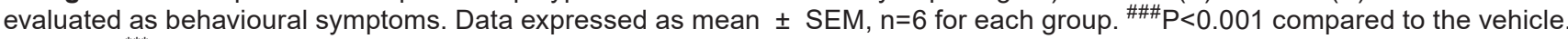
${ }^{* * *} \mathrm{P}<0.001$ compared with Autistic group. (Kolmogorov-Smirnov test, Bonferroni's Multiple Comparison Test). 
A

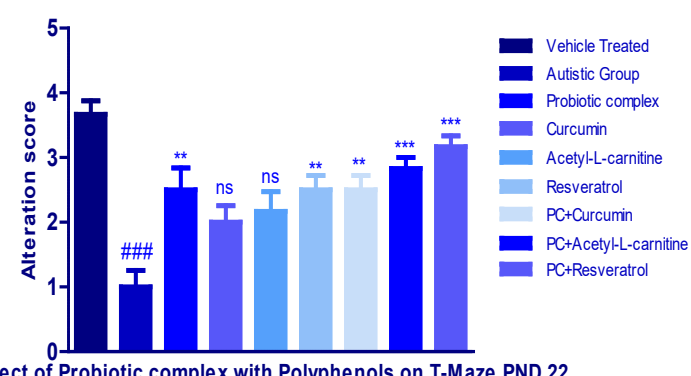

B

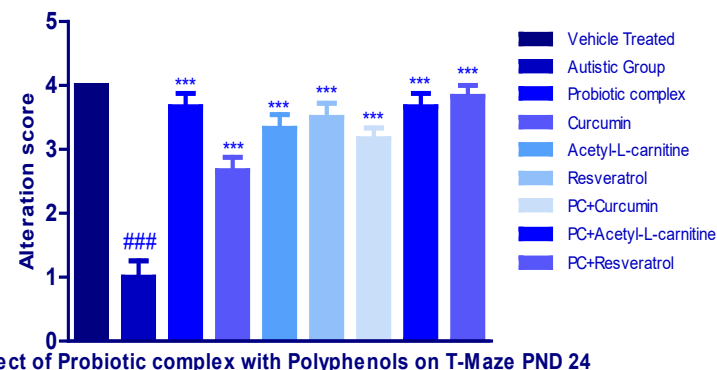

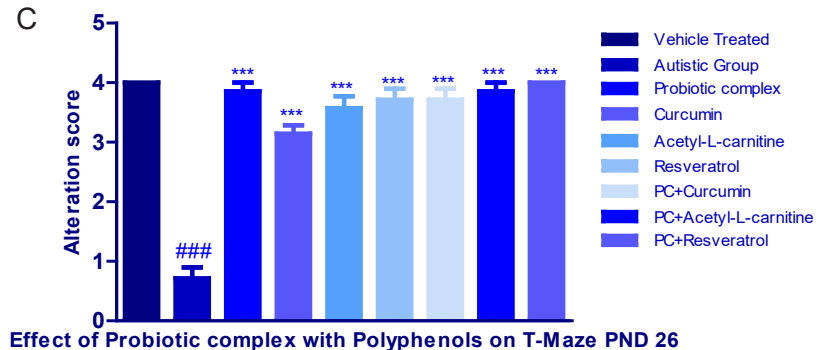

Fig. 2: Effect of probiotic complex with polyphenols combination on T-maze (A) PND 22. (B) PND 24. (C) PND 26 were evaluated as behavioral symptoms. Data expressed as mean \pm SEM, $n=6$ for each group. ${ }^{\# \#} P<0.001$ compared to the vehicle. ${ }^{* * *} \mathrm{P}<0.001$ compared with Autistic group. (Kolmogorov-Smirnov test, Bonferroni's Multiple Comparison Test).

Table 1: Effect of probiotic complex with polyphenols combination on negative geotaxis

\begin{tabular}{|c|c|c|c|c|}
\hline \multicolumn{5}{|c|}{ Negative Geotaxis } \\
\hline Groups & PND 15 & PND 17 & PND 19 & PND 21 \\
\hline Group I (Inulin) & $2.20 \pm 0.37$ & $1.80 \pm 0.37$ & $2.20 \pm 0.58$ & $2.60 \pm 0.60$ \\
\hline Group II (Autistic group) & $13.80 \pm 0.37^{\# \# \#}$ & $13.40 \pm 0.50^{\# \#}$ & $14.80 \pm 0.58^{\# \# \#}$ & $15.60 \pm 0.67^{\# \# \#}$ \\
\hline Group III (Probiotic complex) & $5.60 \pm 0.24^{* * *}$ & $4.80 \pm 0.37^{* * *}$ & $4.40 \pm 0.50^{* * *}$ & $2.80 \pm 0.37^{\star * \star}$ \\
\hline Group IV (Curcumin) & $9.80 \pm 0.58^{* \star *}$ & $7.00 \pm 0.31^{* * *}$ & $5.20 \pm 0.37^{* * *}$ & $3.40 \pm 0.24^{\star * *}$ \\
\hline Group V (Acetyl-L-carnitine) & $11.60 \pm 0.50^{*}$ & $8.20 \pm 0.58^{* * *}$ & $6.40 \pm 0.50^{* * *}$ & $4.00 \pm 0.44^{* * *}$ \\
\hline Group VI (Resveratrol) & $11.40 \pm 0.40^{* *}$ & $8.60 \pm 0.50^{* * *}$ & $6.00 \pm 0.31^{* * *}$ & $3.80 \pm 0.37^{\star * *}$ \\
\hline Group VII (PC + Curcumin) & $5.40 \pm 0.40^{* \star *}$ & $4.40 \pm 0.40^{* * *}$ & $3.00 \pm 0.31^{* * *}$ & $2.20 \pm 0.37^{\star \star \star}$ \\
\hline Group VIII (PC + Acetyl L carnitine) & $5.60 \pm 0.40^{* * *}$ & $4.60 \pm 0.24^{* * *}$ & $4.80 \pm 0.20^{* * *}$ & $2.60 \pm 0.24^{\star * *}$ \\
\hline Group IX (PC + Resveratrol) & $5.80 \pm 0.37^{* \star *}$ & $4.40 \pm 0.24^{* * *}$ & $4.20 \pm 0.20^{\star * *}$ & $2.40 \pm 0.24^{\star \star *}$ \\
\hline
\end{tabular}

Data expressed as mean \pm SEM, $n=6$ for each group. ${ }^{\# \#} \mathrm{P}<0.001$ compared to the vehicle. ${ }^{*} \mathrm{P}<0.05,{ }^{* *} \mathrm{P}<0.01,{ }^{{ }^{* * *}} \mathrm{P}<0.001$ compared with Autistic group. (Kolmogorov-Smirnov test, Bonferroni's Multiple Comparison Test).

Table 2: Effect of probiotic complex with polyphenols combination on memory test (Morris Water Maze)

\begin{tabular}{|c|c|c|c|}
\hline \multicolumn{4}{|c|}{ Morris Water Maze Test } \\
\hline Groups & PND 48 & PND 49 & PND 50 \\
\hline Group I (Inulin) & $53.67 \pm 3.33$ & $39.67 \pm 1.92$ & $15.80 \pm 1.15$ \\
\hline Group II (Autistic group) & $134.2 \pm 6.63^{\# \# \#}$ & $147.4 \pm 9.82^{\# \# \#}$ & $111.8 \pm 7.81^{\# \# \#}$ \\
\hline Group III (Probiotic complex) & $58.80 \pm 3.33^{\star * *}$ & $28.50 \pm 3.35^{\star \star *}$ & $12.66 \pm 1.43^{* * *}$ \\
\hline Group IV (Curcumin) & $64.00 \pm 1.89^{* * *}$ & $26.17 \pm 1.85^{\star * *}$ & $11.33 \pm 0.84^{* * *}$ \\
\hline Group V (Acetyl-L-carnitine) & $60.60 \pm 1.965^{\star * *}$ & $25.67 \pm 0.76^{* \star *}$ & $11.17 \pm 0.90^{* * *}$ \\
\hline Group VI (Resveratrol) & $61.00 \pm 1.897^{* * *}$ & $25.17 \pm 1.24^{* \star \star}$ & $12.83 \pm 1.07^{* * *}$ \\
\hline Group VII (PC + Curcumin) & $60.60 \pm 1.69^{* * *}$ & $24.50 \pm 1.94^{* * *}$ & $9.167 \pm 0.87^{* * *}$ \\
\hline Group VIII (PC + Acetyl-L-carnitine) & $57.40 \pm 1.22^{\star \star \star}$ & $22.33 \pm 2.108^{* * *}$ & $9.00 \pm 0.89^{* \star *}$ \\
\hline Group IX (PC + Resveratrol) & $59.00 \pm 1.87^{* * *}$ & $23.00 \pm 1.82^{* * *}$ & $9.33 \pm 0.71^{* * *}$ \\
\hline
\end{tabular}

Data expressed as mean \pm SEM, $n=6$ for each group. ${ }^{\# \# \#} \mathrm{P}<0.001$ compared to the vehicle. ${ }^{*} \mathrm{P}<0.05,{ }^{* *} \mathrm{P}<0.01,{ }^{* * *} \mathrm{P}<0.001$ compared with Autistic group. (Kolmogorov-Smirnov test, Bonferroni's Multiple Comparison Test). 
vehicle group on 36-40 PND. Treatment with polyphenols alone, probiotic complex and combination of polyphenols with probiotic complex has significantly $(P<0.001)$ shown improvement in all social interaction activities when compared with the autistic group. Among the polyphenols, a minimum latency for identification was observed in the animals treated with curcumin alone and among the combination, probiotic complex with curcumin has shown significantly increased social activity (Table 3).

\subsection{Biochemical Parameters}

\subsubsection{Estimation of IL-6 and TNF- a}

Prenatal induction of autism resulted in significant $(P<0.001)$ increase in IL-6 and TNF- $\alpha$ levels when compared with the control group. Treatment with polyphenols alone, probiotic complex and combination of polyphenols with probiotic complex has significantly $(P<0.001)$ attenuated the increased IL- 6 and TNF- $\alpha$ levels when compared with the autistic group. Among the polyphenols, animals treated with curcumin alone have shown a much-decreased level and among the combi- nation, probiotic complex with curcumin has shown significantly decreased levels (Table 4).

\subsubsection{Estimation of BDNF}

Significantly $(P<0.001)$ elevated levels of BDNF levels were seen in autistic group when compared with the vehicle group. Treatment with polyphenols alone, probiotic complex and combination of polyphenols with probiotic complex has significantly $(P<0.001)$ ameliorated the BDNF levels when compared with the autistic group. Among the polyphenols, animals treated with resveratrol alone have shown effective decreased level and among the combination, probiotic complex with curcumin has shown significantly decreased levels (Table 5).

\subsubsection{Estimation of 5-HT}

Autistic rats have shown significant $(P<0.001)$ hypersertonomia when compared with the vehicle group. Treatment with polyphenols alone, probiotic complex and combination of polyphenols with probiotic complex has significantly $(P<0.001)$ decreased serotonin levels when

Table 3: Effect of probiotic complex with polyphenols combination on social interaction

\begin{tabular}{|c|c|c|c|c|c|}
\hline \multicolumn{6}{|c|}{ Social Interaction } \\
\hline Groups & Allogrooming & $\begin{array}{l}\text { Anogenital } \\
\text { inspection }\end{array}$ & Pinnings & Play behavior & Social exploration \\
\hline Group I (Inulin) & $13.17 \pm 0.65$ & $29.17 \pm 1.30$ & $22.83 \pm 1.62$ & $9.83 \pm 0.60$ & $22.00 \pm 0.57$ \\
\hline Group II (Autistic group) & $3.00 \pm 0.51 \# \# \#$ & $8.00 \pm 1.065 \# \# \#$ & $7.83 \pm 1.19 \# \# \#$ & $1.33 \pm 0.33 \# \# \#$ & $6.83 \pm 0.74 \# \#$ \\
\hline Group III (Probiotic complex) & $13.33 \pm 0.55^{\star * \star}$ & $25.67 \pm 0.76$ & $22.50 \pm 0.76^{\star * *}$ & $11.67 \pm 0.42^{\star * *}$ & $23.33 \pm 0.66^{* * *}$ \\
\hline Group IV (Curcumin) & $9.83 \pm 0.30^{\star \star \star}$ & $25.00 \pm 0.36^{\star * \star}$ & $23.00 \pm 0.57^{\star \star *}$ & $9.167 \pm 0.30^{* * *}$ & $22.83 \pm 0.79^{* * *}$ \\
\hline Group V (Acetyl-L-carnitine)\ & $9.66 \pm 0.33^{* * *}$ & $26.67 \pm 0.49^{* * *}$ & $22.00 \pm 1.09^{\star * *}$ & $9.667 \pm 0.55^{\star \star \star}$ & $25.00 \pm 0.44^{* * *}$ \\
\hline Group VI (Resveratrol) & $10.33 \pm 0.71^{* * *}$ & $25.67 \pm 0.42^{\star *}$ & $23.17 \pm 0.79^{\star \star \star}$ & $11.83 \pm 0.47^{\star \star \star}$ & $24.17 \pm 0.47^{* * *}$ \\
\hline Group VII (PC + Curcumin) & $12.33 \pm 0.33^{* * *}$ & $27.17 \pm 0.47^{\star \star \star}$ & $25.17 \pm 0.60^{\star \star *}$ & $12.67 \pm 0.49^{\star \star \star}$ & $24.83 \pm 0.47^{* * *}$ \\
\hline Group VIII (PC + Acetyl-L-carnitine) & $12.17 \pm 0.40^{* * *}$ & $28.83 \pm 0.60^{\star * *}$ & $26.50 \pm 1.02^{\star \star *}$ & $13.17 \pm 0.47^{\star * \star}$ & $27.83 \pm 0.60^{\star * *}$ \\
\hline Group IX (PC + Resveratrol) & $11.17 \pm 0.47^{* * *}$ & $28.17 \pm 0.60^{\star * *}$ & $26.33 \pm 0.42^{* * *}$ & $14.83 \pm 0.65^{\star * *}$ & $25.50 \pm 0.42^{* * *}$ \\
\hline
\end{tabular}

Data expressed as mean \pm SEM, $n=6$ for each group. ${ }^{\# \#} \mathrm{P}<0.001$ compared to the vehicle. ${ }^{*} \mathrm{P}<0.05,{ }^{* *} \mathrm{P}<0.01,{ }^{* * *} \mathrm{P}<0.001$ compared with Autistic group. (Kolmogorov-Smirnov test, Bonferroni's Multiple Comparison Test).

Table 4: Effect of probiotic complex with polyphenols combination on proinflammatory cytokines levels

\begin{tabular}{lll}
\hline & Proinflammatory Cytokines & \\
\hline Groups & $I L-6(p g / m l)$ & $T N F-\alpha(p g / m l)$ \\
\hline Group I (Inulin) & $26.78 \pm 1.14$ & $90.80 \pm 2.68$ \\
Group II (Autistic group) & $47.58 \pm 1.07^{\# \#}$ & $134.61 \pm 1.85^{\text {\#\# }}$ \\
Group III (Probiotic complex) & $27.35 \pm 1.28^{* * *}$ & $100.1 \pm 1.56^{* * *}$ \\
Group IV (Curcumin) & $28.52 \pm 0.17^{* * *}$ & $108.1 \pm 1.712^{* * *}$ \\
Group V (Acetyl-L-carnitine) & $34.95 \pm 0.69^{* * *}$ & $113.8 \pm 2.311^{* * *}$ \\
Group VI (Resveratrol) & $30.74 \pm 0.36^{* * *}$ & $112.6 \pm 1.808^{* * *}$ \\
Group VII (PC + Curcumin) & $27.10 \pm 0.36^{* * *}$ & $97.87 \pm 20.81^{* * *}$ \\
Group VIII (PC + Acetyl-L-carnitine) & $30.90 \pm 0.83^{* * *}$ & $107.2 \pm 1.15^{* * *}$ \\
Group IX (PC + Resveratrol) & $27.96 \pm 0.42^{* * *}$ & $104.6 \pm 0.99^{* * *}$
\end{tabular}

Data expressed as mean $\pm \mathrm{SEM}, \mathrm{n}=6$ for each group. ${ }^{\# \#} \mathrm{P}<0.001$ compared to the vehicle. ${ }^{*} \mathrm{P}<0.05,{ }^{* *} \mathrm{P}<0.01,{ }^{*{ }^{* *}} \mathrm{P}<0.001 \mathrm{compared}$ with Autistic group. (Kolmogorov-Smirnov test, Bonferroni's Multiple Comparison Test).। 
compared with the autistic group. Among the polyphenols, animals treated with resveratrol alone have shown effective decreased level and among the combination, probiotic complex with resveratrol has shown significantly decreased levels (Table 5).

\subsubsection{Effect of Probiotic Strains on AChE Levels}

In the autistic group, significant $(P<0.001)$ increased acetylcholinesterase activity was seen when compared with the control group. Treatment with polyphenols alone, probiotic complex and combination of polyphenols with probiotic complex has significantly $(P<0.001)$ attenuated the increased enzyme activity when compared with the autistic group. Among the polyphenols, animals treated with acetyl-l-carnitine alone have shown effective decreased level and among the combination, probiotic complex with acetyl-1-carnitine has shown significantly decreased levels (Table 5).

Table 5: Effect of probiotic complex with polyphenols combination on BDNF, 5-HT, AChE

\begin{tabular}{|c|c|c|c|}
\hline \multicolumn{4}{|c|}{$B D N F, 5-H T, A C h E$} \\
\hline Groups & $B D N F(p g / m l)$ & $5-H T(n g / m l)$ & $\operatorname{AchE}(\mu \mathrm{M} / \mathrm{min} / \mathrm{mg}$ tissue $)$ \\
\hline Group I (Inulin 3mg, p.o daily) & $45.12 \pm 1.05$ & $4.98 \pm 0.27$ & $1.58 \pm 0.15$ \\
\hline Group II (Autistic group) & $59.74 \pm 1.07^{\# \# \#}$ & $26.84 \pm 1.31^{\# \# \#}$ & $6.40 \pm 0.36^{\# \#}$ \\
\hline Group III (Probiotic complex) & $45.57 \pm 1.06^{* * *}$ & $8.39 \pm 1.74^{* * *}$ & $1.64 \pm 0.12^{* * *}$ \\
\hline Group IV (Curcumin) & $47.47 \pm 0.27^{* * *}$ & $9.56 \pm 0.20^{\star * *}$ & $1.66 \pm 0.05^{* * *}$ \\
\hline Group V (Acetyl-L-carnitine) & $48.28 \pm 0.63^{* * *}$ & $10.22 \pm 0.28^{* * *}$ & $1.63 \pm 0.01^{* * *}$ \\
\hline Group VI (Resveratrol) & $46.83 \pm 0.31^{* * *}$ & $9.00 \pm 0.24^{\star * *}$ & $1.69 \pm 0.014^{* * *}$ \\
\hline Group VII (PC + Curcumin) & $45.26 \pm 0.55^{\star * *}$ & $7.84 \pm 0.28^{* * *}$ & $1.60 \pm 0.05^{* * *}$ \\
\hline Group VIII (PC + Acetyl-L-carnitine) & $46.14 \pm 0.50^{* * *}$ & $9.02 \pm 0.38^{\star \star *}$ & $1.54 \pm 0.02^{\star \star \star}$ \\
\hline Group IX (PC + Resveratrol) & $45.96 \pm 0.58^{* * *}$ & $6.24 \pm 0.35^{\star \star *}$ & $1.63 \pm 0.03^{* * *}$ \\
\hline
\end{tabular}

Data expressed as mean \pm SEM, $n=6$ for each group. ${ }^{\# \#} \mathrm{P}<0.001$ compared to the vehicle. ${ }^{*} \mathrm{P}<0.05,{ }^{* *} \mathrm{P}<0.01,{ }^{* * *} \mathrm{P}<0.001$ compared with Autistic group. (Kolmogorov-Smirnov test, Bonferroni's Multiple Comparison Test).

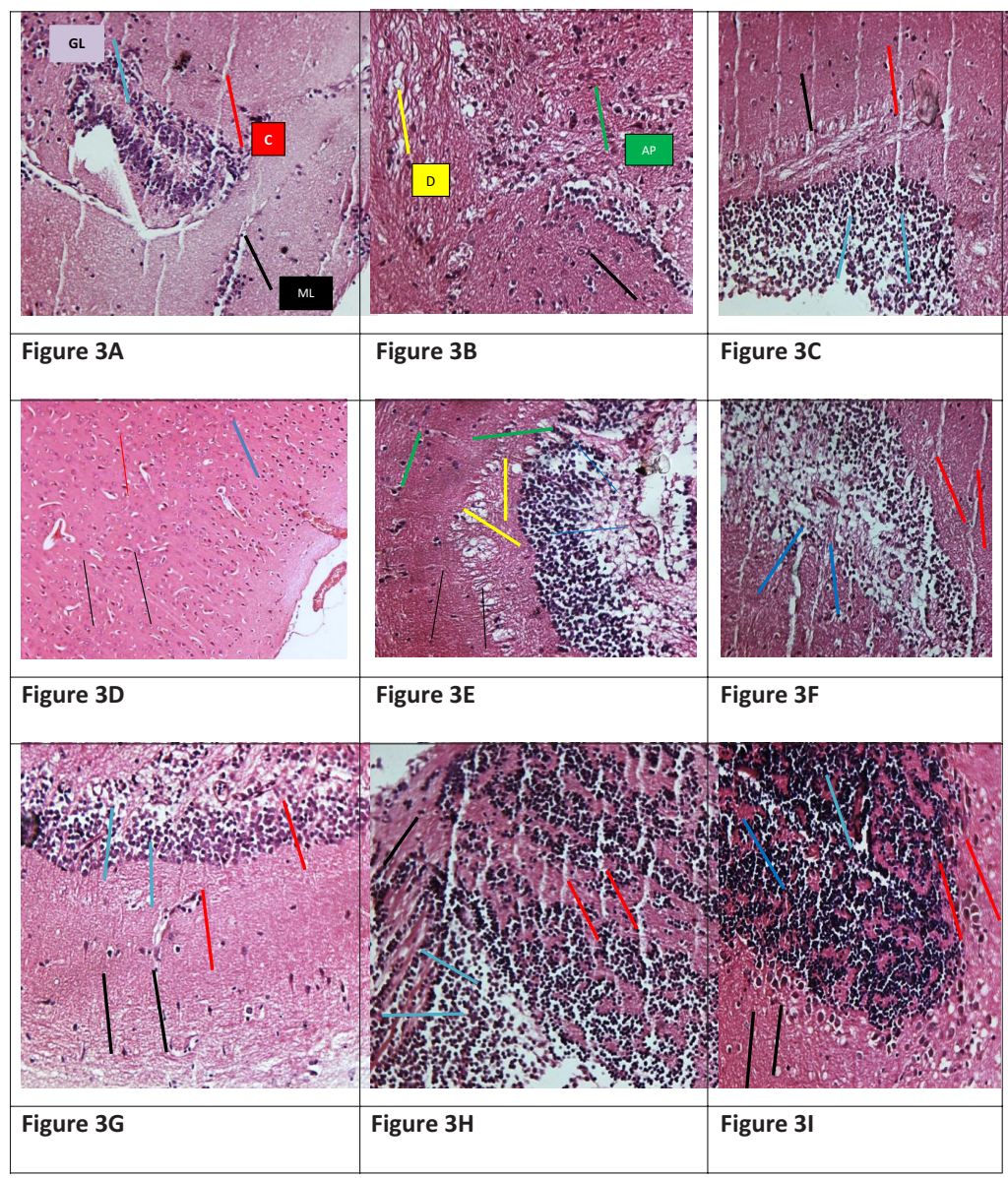

Fig. 3: Effect of Probiotic complex with polyphenols combination on histopathology of cerebellum. 


\section{HISTOPATHOLOGY}

Histopathology of brain areas has revealed normal molecular layer and cerebellum areas in vehicle-treated animals whereas damaged neurons due to apoptosis were observed in animals treated with valproic acid. The animals treated with polyphenols alone, probiotic complex and polyphenols with probiotic complex have shown a normal molecular level of cerebellum with mild degeneration and recovering stages of neurons.Figure 3(A) Vehicle treated group, 3(B) Autistic group, 3(C) Probiotic complex, 3(D) Curcumin, 3(E) Acetyl-1carnitine, 3(F) Resveratrol, 3(G) PC + Curcumin, 3(H) PC + Acetyl-l-carnitine, 3(I) PC + Resveratrol; Black arrow: Normal molecular layer of cerebellum; Red arrow: Capillary neurons appeared normal; Blue arrow: Normal granular layer of cerebellum; Green arrow: Apoptosis of many neurons; Yellow arrow: Damaged neurons.

\section{DISCUSSION}

The adjustment of the microbiota-gut-brain alignment with a perspective on forestalling and treating ASD has emerged as a center of research for the present scientific community. The mutual correlation between autism and changes in the microbiota has been affirmed by numerous animal and human studies. ${ }^{31}$ Literature has suggested the use of probiotics and phytopharmaceuticals (polyphenols and acetyl-1-carnitine) which have crucial roles in improving the behavioral conditions of ASDs. 9,32

The present research study utilized the pre-natal model of autism in which rats exhibited impaired behavior associated with autistic people. ${ }^{33}$ The current investigation evaluated the probiotic complex and polyphenols alone and combination for improving the conditions of autism and alteration of gut bacteria. Outright risk and the hazard ratio (HR) of autism spectrum disorder and childhood autism in children after introduction of valproate in pregnancy have previously been demonstrated. ${ }^{34}$ Various behavioral parameters have been assessed to measure the social and cognitive variations with polyphenols and probiotic complex (L. acidophilus, L. rhamnosus, B. longum, B. bifidum, $S$. thermophiles and $S$. boulardii).

In this model autistic rats have generally exerted gut problems such as lack of interest in eating food, irregular bowel movements, weight loss, fatigue, alopecia and sometimes hyperactive behavior. All these gut-related problems were recovered well in probiotic complex treatment.

The opening of eye was observed to be postponed in animals subjected to valproic acid alone on 12-16 PND as also observed by Gottfried et al., 2013 in his studies to develop demonstrative animal model for autism by exposing animals to valproic acid. ${ }^{10}$ Conversely, usual eye-opening was seen in treatment groups. Comparative outcomes were obtained from the studies conducted on autism with curcumin by Bhandari \& Kuhad, 2015; acetylL-carnitine by Geethakrishnan et al., 2019 and resveratrol Tekula et al., 2018. In the present study, combination of probiotic complex and resveratrol has shown highly significant improvement.

In the T-maze test, the absence of impulsive variations was observed in the autistic group. In the treatment groups, the variation scores were restored to normal with an enhanced activity seen in animals treated with probiotic complex with resveratrol combination.

Negative geotaxis is used to evaluate vestibular function and motor performance of cerebellar development and function. ${ }^{27}$ Increase in the time taken to reorient on the slanted plane was found in the autistic group. Reorientation time was restored in treatment groups from 15 PND onwards. The results were in accordance with the studies performed by Tekula et al., 2018 and Geethakrishnan et al., 2019. Probiotic complex with curcumin combination has shown highly significant score.

Morris's water maze was utilized to assess the psychological and cognitive function of an individual. ${ }^{37}$ In autistic group, delayed latency for identification of hidden platform was seen. Among all treatment groups, probiotic complex with acetyl-l-carnitine has shown improved steering ability and memory performance might be because of the cognition-enhancing effects.

One of the defects in autistic subjects is a discrepancy in social interaction owing to the inability to recognize and communicate signals in the brain. ${ }^{38}$ Among all groups combination of probiotic complex with curcumin has improved social behavior which is attributed to its gut-brain communication. Results here obtained are in confined with Bhandari \& Kuhad, 2015; acetyl-L-carnitine by Geethakrishnan et al., 2019; resveratrol Tekula et al., 2018 and Schiavi et al., 2019.

The biochemical studies have revealed elevated levels of IL-6, TNF- $\alpha$, BDNF, 5-HT and AChE levels in autistic subjects. IL-6 activates cellular responses to inflammation, neuronal growth \& persistence, myelination and demyelination. ${ }^{40}$ In the present study, higher concentrations of IL- 6 and TNF- $\alpha$ in the cerebellum was seen in autistic rats. In the treatment groups there is significant decrease in the proinflammatory cytokines by their immune supportive mechanisms. Specifically treatment probiotic complex with curcumin combination has given superiority in recovery of elevated proinflammatory cytokines. 
BDNF is a neurotrophic factor (NF) that helps in the growth and endurance of neurons. ${ }^{41}$ Increase in BDNF levels in autistic subjects was observed. In the treatment group's good correction of BDNF levels was seen after ELISA estimation. In comparison other groups, probiotic complex with curcumin has given significant results.

During CNS development, 5-HT plays important role in the regulation of neuronal differentiation and migration, myelination and synapse formation. ${ }^{42}$ Increased serum 5-HT levels were seen in the autistic group resulting in behavioral abnormality. Treatment with poly phenols alone, probiotic complex and in combination have decreased 5-HT levels, among all groups probiotic complex with resveratrol combination has good recovery in elevated 5-HT.

In the CNS, acetylcholine is concerned with the learning and memory process, including attention, cognitive function, social interactions and stereotypical behaviors. ${ }^{43}$ VPA exposure reduces the concentration of acetylcholine in autistic animals. Among all study groups, significant results have been with probiotic complex with acetyl-1-carnitine in decreasing AChE levels.

Histopathology of the cerebellum revealed neuronal degeneration and apoptosis in the autistic group as indicated by the VPA triggered oxidative stress and degeneration. Through this study it is understood that rather than alone treatment, combination treatments are significantly amended the VPA induced degeneration of neurons and apoptosis in cerebellum. Although treatment significant $\mathrm{PC}+$ Curcumin, $\mathrm{PC}+$ Reseveratrol are superior in protecting the VPA degeneration in neurons, might be due to their hierarchy in therapeutic efficacy.

\section{CONCLUSION}

With our study, it is emphasized that probiotic complex with polyphenols (curcumin and resveratrol) and acetyl1-carnitine combination is synergetic, their combination augmented the modulation of the dysbiotic gut, supported the gut-brain-axis. With the right duration of treatment, it is observed that maximum recovery of autistic symptoms has been seen. These functional foods (probiotics and phytochemicals) can be able to deliver more targeted benefits in multi etiological conditions. Both the combination of probiotic complex with polyphenols are advisable, mutually they benefit each other and establishes symbiotic relation with gut, making healthier gut and amends the autism and its associated dysbiotic conditions. The advice of this combination is a novel approachable therapy for the safer and secure treatment in autism. This combination has given diversified benefits; recovery of autistic conditions is maximum as previously explained.

\section{ACKNOWLEDGMENT}

We are sincerely thankful to UNIQUE BIOTECH LTD. for their kind support in providing probiotic strains. Also, we express sincere gratitude towards Chairman - Dr. Palla Rajeshwar Reddy, Dean - Dr. Vasudha Bakshi, School of Pharmacy, and Anurag University for their support.

\section{REFERENCES}

1. Eloe-Fadrosh EA, Rasko DA. The Human microbiome: from symbiosis to pathogenesis. Annu. Rev. Med 2013;64:145-163. https://doi.org/10.1146/annurev-med-010312-133513.

2. Abdellatif B, McVeigh C, Bendriss G, Chaari A. 2020. The Promising role of probiotics in managing the altered gut in autism spectrum disorders. IJMS 2020;21:4159. https://doi. org/10.3390/ijms21114159

3. Posar A, Resca F, Visconti P. Autism according to diagnostic and statistical manual of mental disorders 5thedition: The need for further improvements. J Pediatr Neurosci 2015;10:146. https://doi.org/10.4103/1817-1745.159195

4. Elsabbagh M, Divan G, Koh YJ, Kim YS, Kauchali S, Marcín $\mathrm{C}$, et al. Global prevalence of autism and other pervasive developmental disorders. Autism Res 2012;5:160-179. https:// doi.org/10.1002/aur.239

5. Faras H, Al Ateeqi N, Tidmarsh L. Autism spectrum disorders. Annals of Saudi Medicine 2010;30:295-300. https://doi. org/10.4103/0256-4947.65261

6. McPartland J, Volkmar F.R. 2012. Autism and related disorders. Hand book of clinical neurology 2012:407-418. https:// doi.org/10.1016/b978-0-444-52002-9.00023-1

7. Holingue C, Newill C, Lee LC, Pasricha PJ, Daniele Fallin M. Gastrointestinal symptoms in autism spectrum disorder: A review of the literature on ascertainment and prevalence. Autism Research 2017;11:24-36. https://doi.org/10.1002/ aur.1854

8. Azhari A, Azizan F, Esposito G. A systematic review of gutimmune-brain mechanisms in Autism Spectrum Disorder. Dev Psychobiol 2018;61:752-771. https://doi.org/10.1002/ dev.21803

9. $\mathrm{Ng}$ Q, Loke W, Venkatanarayanan N, Lim D, Soh A, Yeo W. A Systematic review of the role of prebiotics and probiotics in autism spectrum disorders. Medicina 2019;55:129. https:// doi.org/10.3390/medicina55050129

10. Gottfried C, Victorio BJ, Diego B, Geancarlo Z, Roberta B, Tamara V, et al. Valproic acid in autism spectrum disorder: from an environmental risk factor to a reliable animal model. Recent Advances in Autism Spectrum Disorders 2013:1.

11. Bölte S, Girdler S, Marschik PB, 2018. The contribution of environmental exposure to the etiology of autism spectrum disorder. Cell. Mol. Life Sci 2018;76:1275-1297. https://doi. org/10.1007/s00018-018-2988-4

12. Sivamaruthi BS, Suganthy N, Kesika P, Chaiyasut C. The Role of microbiome, dietary supplements, and probiotics in autism spectrum disorder. IJERPH 2020;17:2647. https://doi. org/10.3390/ijerph17082647

13. Serra D, Almeida LM, Dinis TCP. Polyphenols as food bioactive compounds in the context of Autism Spectrum 
Disorders: A critical mini-review. Neuroscience \& Biobehavioral Reviews 2019 102:290-298. https://doi.org/10.1016/j. neubiorev.2019.05.010

14. Sharma P, Kumar A, Singh D. Dietary flavonoids interaction with CREB-BDNF Pathway: An Unconventional Approach for Comprehensive Management of Epilepsy. Current neuropharmacology 2019; 17:1158-1175. https://doi.org/10.2 174/ $1570159 X 17666190809165549$

15. Demarquoy C, Demarquoy J. Autism and carnitine: A possible link. WJBC. 2019;10: 7-16. https://doi.org/10.4331/wjbc.v10.i1.7

16. Ma Y, Liu T, Fu J, Fu S, Hu C, Sun B, et al. Lactobacillus acidophilus exerts neuroprotective effects in mice with traumatic brain injury. J Nutr 2019;149:1543-1552. doi: 10.1093/jn/ nxz105.

17. Savignac HM, Tramullas M, Kiely B, Dinan TG, Cryan JF.. Bifidobacteria modulate cognitive processes in an anxious mouse strain. Behavioural Brain Research 2015; 287:59-72. doi:10.1016/j.bbr.2015.02.044

18. Maldonado Galdeano C, Cazorla SI, Lemme Dumit JM, Vélez E, Perdigón G. Beneficial effects of probiotic consumption on the immune system. Ann Nutr Metab 2019. 74, 115-124. https:// doi.org/10.1159/000496426

19. Dargahi N, Matsoukas J, Apostolopoulos V, 2020. Streptococcusthermophilus ST285 alters pro-inflammatory to antiinflammatory cytokine secretion against multiple sclerosis peptide in mice. Brain sciences 2020;10:126. https://doi.org/10 3390/brainsci10020126

20. Umbrello G, Esposito S. Microbiota and neurologic diseases: potential effects of probiotics. J Transl Med 2016:14. https:// doi.org/10.1186/s12967-016-1058-7

21. Bhandari R, Kuhad A. Neuropsychopharmacotherapeutic efficacy of curcumin in experimental paradigm of autism spectrum disorders. Life Sciences. 2015;141:156-169. https:// doi.org/10.1016/j.lfs.2015.09.012

22. Xie W, Ge X, Li L, Yao A, Wang X, Li M, et al, 2018. Resveratrol ameliorates prenatal progestin exposure-induced autism-like behavior through ER $\beta$ activation. Molecular Autism 2018: 9. https://doi.org/10.1186/s13229-018-0225-5

23. Malaguarnera M, Cauli O 2019. Effects of 1-carnitine in patients with autism spectrum disorders: review of clinical studies. Molecules 2019;24:4262. https://doi.org/10.3390/ molecules 24234262

24. Liu F, Horton-Sparks K, Hull V, Li RW, Martínez-Cerdeño V. The valproic acid rat model of autism presents with gut bacterial dysbiosis similar to that in human autism. Molecular Autism 2018:9. https://doi.org/10.1186/s13229-0180251-3

25. Falck-Ytter T, Bölte S, Gredebäck G. Eye tracking in early autism research. J Neurodevelop Disord 2013;5. https: //doi.org/10.1186/1866-1955-5-28

26. Hrabovska SV, Salyha YuT. 2016. Animal models of autism spectrum disorders and behavioral techniques of their examination. Neurophysiology 2016;48:380-388. https://doi. org/10.1007/s11062-017-9613-2

27. Ruhela RK, Soni S, Sarma P, Prakash A, Medhi B. 2019. Negative geotaxis: An early age behavioral hallmark to VPA rat model of autism. Annals of Neurosciences 2019;26:25-31. https://doi.org/10.5214/ans.0972.7531.260106
28. Silverman JL, Yang M, Lord C, Crawley JN. Behavioural phenotyping assays for mouse models of autism. Nat Rev Neurosci 2010;11:490-502. https://doi.org/10.1038/nrn 2851

29. La Fratta I, Tatangelo R, Campagna G, Rizzuto A, Franceschelli S, Ferrone A, et al. 2018. The plasmatic and salivary levels of IL-1 $\beta$, IL-18 and IL- 6 are associated to emotional difference during stress in young male. Sci Rep 2018:8. https:// doi.org/10.1038/s41598-018-21474-y

30. Paradiso B, Simonato M, Thiene G, Lavezzi A. From fix to fit into the autoptic human brains. Eur J Histochem 2018;62:3. https://doi.org/10.4081/ejh.2018.2944

31. Srikantha P, Mohajeri MH, 2019. The Possible Role of the Microbiota-Gut-Brain-Axis in Autism Spectrum Disorder IJMS 2019;20:2115. https://doi.org/10.3390/ijms20092115

32. Kumar Singh A, Cabral C, Kumar R, Ganguly R, Kumar Rana H, Gupta A, et al. 2019. Beneficial effects of dietary polyphenols on gut microbiota and strategies to improve delivery efficiency. Nutrients 2019;11:2216. https://doi.org/10.3390/ nu11092216

33. Hirsch MM, Deckmann I, Fontes-Dutra M, Bauer-Negrini G, Nunes G,D, Nunes W, et al. Behavioral alterations in autism model induced by valproic acid and translational analysis of circulating microRNA. Food and Chemical Toxicology 2018;115:336-343

34. Christensen J, Grønborg TK, Sørensen MJ, Schendel D, Parner ET, Pedersen LH, et al. 2013. Prenatal valproate exposure and risk of autism spectrum disorders and childhood autism. JAMA 2013;309:1696. https://doi.org/10.1001/ jama.2013.2270

35. Geethakrishnan S, Bakshi V, Katta S, Gandhamalla P, Kaluwala M, Devarakonda K, et al. Ameliorative effect of acetyl-l-carnitine in valproic acid induced autistic albino rats: prenatal model. Journal of the Neurological Sciences 2019;405: 116-117.

36. Tekula MR, Sunand K, Begum N, Kakalij RM, Bakshi V. 2018. Neuroprotective effect of resveratrol on valproic acid induced oxidative stress autism in swiss albino mice. Int J Pharm Sci Drug Res 2018;10:103-10. doi:10.25004/IJPSDR.2018. 100301.

37. Schneider T, Przewłocki R. Behavioral alterations in rats prenatally exposed to valproic acid: animal model of autism. Neuropsychopharmacol 2004;30:80-89. https://doi. org/10.1038/sj.npp.1300518

38. Mayer EA, Padua D, Tillisch K, 2014. Altered brain-gut axis in autism: comorbidity or causative mechanisms?. BioEssays 2014;36(10):933-939. https://doi.org/10.1002/bies.201400075

39. Schiavi S, Iezzi D, Manduca A, Leone S, Melancia F, Carbone $\mathrm{C}$, et al. reward-related behavioral, neurochemical and electrophysiological changes in a rat model of autism based on prenatal exposure to valproic acid. Frontiers in cellular neuroscience 2019;13: 479. https://doi.org/10.3389/fncel.2019.00479

40. Myers SM, Johnson CP. American Academy of Pediatrics Council on Children with Disabilities. Management of children with autism spectrum disorders. Pediatrics 2007;120: 1162-1182. https://doi.org/10.1542/peds.2007-2362

41. Kasarpalkar NJ, Kothari ST, Dave UP. Brain-Derived Neurotrophic Factor in children with autism spectrum disorder. ANS 2014: 21. https://doi.org/10.5214/ ans.0972.7531.210403 
42. Ragozzino ME, Pal SN, Unick K, Stefani MR, Gold PE. Modulation of hippocampal acetylcholine release and spontaneous alternation scores by intrahippocampal glucose injections. The Journal of neuroscience 1998;18(4):15951601. https://doi.org/10.1523/JNEUROSCI.18-04-01595. 1998

43. Ming X, Cheh MA, Yochum CL, Halladay AK, Wagner GC. Evidence of oxidative stress in autism derived from animal models. American J. of Biochemistry and Biotechnology 2008;4:218-225. https://doi.org/10.3844/ajbbsp.2008. 218.225
How to cite this article: Sunand K, Mohan GK, Bakshi V. Synergetic Potential of Combination Probiotic Complex with Phytopharmaceuticals in Valproic Acid Induced Autism: Prenatal Model. Int. J. Appl. Pharm. Sci. Res. (2021);6(3): 33-43. doi: https://doi. org/10.21477/ijapsr.6.3.02

Source of Support: Nil.

Conflict of Support: None declared. 